\title{
Timing Skew Compensation Methods for CMOS SPAD Line Sensors Used for Raman Spectroscopy
}

\author{
Tuomo Talala, Ilkka Nissinen \\ Circuits and Systems Research Unit, University of Oulu, Oulu, Finland \\ firstname.lastname@oulu.fi
}

\begin{abstract}
Two methods were developed to compensate for the timing skew of CMOS SPAD line sensors used for time-resolving Raman spectroscopy. Both methods were tested using a timeresolving Raman spectrometer built around a 256-channel CMOS SPAD line sensor. As an example, Raman spectrum of highly fluorescent sesame seed oil was measured. Most of the distortion in the measured spectrum was caused by the timing skew and about $75 \%$ of it could be removed by using either of the methods presented.
\end{abstract}

Keywords—Raman spectroscopy; time gating; timing skew

\section{INTRODUCTION}

One of the weaknesses of Raman spectroscopy is its limited use for measuring highly fluorescent samples. Strong fluorescence can mask weak Raman scattering, and it can be difficult and time-consuming to measure the Raman spectrum of samples that have high fluorescence levels. Many techniques have been developed to overcome this problem: time gating, photobleaching and surface-enhanced Raman spectroscopy to name a few [1-3].

Recently time-gated Raman spectrometers have been built around single-photon avalanche diodes (SPAD) manufactured in CMOS (complementary metal-oxide-semiconductor) technology $[4,5]$. This is a compact and cost-effective solution because it is possible to integrate electronics for time-resolving photon counting and the SPADs in a single CMOS chip. In the latest CMOS SPAD line sensors for time-correlated single photon counting, the resolution of time-to-digital converters (TDC) is about $50 \mathrm{ps}[6,7]$.

While time-correlated single photon counting is a practical way to implement time gating for fluorescence suppression in Raman spectroscopy, the use of a multichannel line sensor itself creates a new challenge. All channels of the sensor are never exactly identical and mismatch between the channels will cause variation on the time gating along the spectral range, causing distortion to the spectrum. Fig. 1 shows an example where the endpoint of the time gate is at $1200 \mathrm{ps}$ for channel A and at 1222 ps for channel B, and Raman scattering is observed only at the wavelength of channel $\mathrm{A}$. The number of fluorescence photons detected within 750-1200 ps is same for both channels. In addition, channel A detects Raman photons and channel B detects extra fluorescence photons within 1200-1222 ps. In total, both channels detect the same number of photons and no difference is observed between the channels even if Raman photons were detected only by channel A. The case shown in Fig. 1 corresponds to a sample that has a fluorescence lifetime of $2 \mathrm{~ns}$ and a fluorescence-to-Raman ratio of 100. It is obvious

This work was supported by Academy of Finland under Contracts 314404 and 292609. that for such sample, a variation between the endpoints of the time gate for channels nearby should be a lot smaller than $22 \mathrm{ps,}$ or the Raman spectrum can not be measured. In applications dealing with samples that have a higher fluorescence-to-Raman ratio or a shorter fluorescence lifetime, even higher level of uniformity is required.

Since no sensor has ideal timing performance, the result of a time-gated measurement is always somewhat distorted by the timing skew of the sensor. In [6] this distortion was corrected by post-processing, using a certain reference sample that has similar fluorescence level as the actual measurement sample. Timing characterization for a CMOS SPAD line sensor was done in [8], but the results were not used for timing skew compensation. Also, the sensor was characterized in a specific characterization environment, not in its normal operation in a Raman spectrometer [8]. In this paper, two new post-processing methods for timing skew correction are presented. The methods are based on characterization of the sensor's timing properties with a reference sample when the sensor is operating normally in a Raman spectrometer. No characterization environment or sample-specific references are needed. The efficiency of the new post-processing methods was verified with oil sample measurements.

\section{Characterization OF A SENSOR's Timing SKEW}

Two different techniques for the characterization of the timing properties of a line sensor are presented. The first technique gives detailed data about the sensor based on a series of measurements. The second technique only reveals the dominant shape of the timing skew but consists of a single measurement and is therefore very straightforward to use.

\section{A. Precise Method for the Characterization of a Sensor}

This method consists of two completely separate measurements. In the first measurement, the sensor is illuminated with a continuous constant light source, e.g. with a

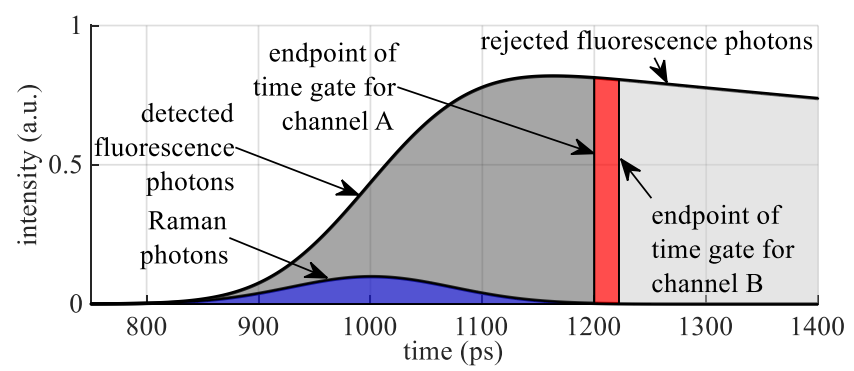

Fig. 1. Variation on the number of detected fluorescence photons caused by mismatch between sensor channels. 
halogen lamp. As the photon count for each TDC bin depends on the intensity of the light and on the bin width, for an ideal sensor having identical bins, the average photon count would be equal for all bins. With a real sensor, the average photon counts vary due to variation of bin widths. Actually, the ratios of photon counts are equal to the ratios of bin widths.

The purpose of the second measurement phase is to set relative bin widths to absolute time scale by measuring an interval between two selected bins A and B. In this phase, a pulsed laser and a reference sample with a short fluorescence lifetime are used. The reference sample should be chosen so that its fluorescence emission covers the whole spectral range of the sensor. Procedure for the second measurement is shown in Fig. 2 a). First, the measurement range of TDC is swept gradually using an adjustable delay element to find the time position of bin A. When the photon count of bin A is plotted against the sweep step as shown in Fig. 2 b), interval $t_{1}$ corresponds to a delay value giving the highest photon count. Then the measurement range of the TDC is delayed for $t_{\mathrm{c}}$ to move the fluorescence emission to the end of the TDC's range and another sweep is made. Again, the photon count of another selected bin B is plotted against the sweep step of the second sweep, and interval $t_{2}$ corresponds to a delay value giving the highest photon count. Assuming that the highest photon count is detected when the peak of the fluorescence emission is in the middle of a bin, the interval between the midpoints of selected bins A and B is

$$
t_{\mathrm{c}}-t_{1}-t_{2}
$$

Let $n_{x}$ be the photon count of bin $x$ in the first (continuous light) measurement phase. Now the number of photons detected in the first measurement between the midpoint of bin $\mathrm{A}$ and the midpoint of bin $B$ is

$$
0.5 n_{\mathrm{A}}+\sum_{i=\mathrm{A}+1}^{\mathrm{B}-1} n_{i}+0.5 n_{\mathrm{B}} .
$$

Since the photon count in (2) is detected during the period in (1), we can define a coefficient $C_{t / n}$ as a ratio of (1) and (2),

$$
C_{t / n}=\frac{t_{\mathrm{c}}-t_{1}-t_{2}}{0.5 n_{\mathrm{A}}+\sum_{i=\mathrm{A}+1}^{\mathrm{B}-1} n_{i}+0.5 n_{\mathrm{B}}} .
$$

As the coefficient $C_{t / n}$ gives a ratio between a bin width and a photon count $n_{x}$, it is possible to calculate every bin width just by multiplying photon count $n_{x}$ with the coefficient $C_{t / n}$. Bins A and $B$ should be chosen to maximize the value of (1) as it makes the coefficient $C_{t / n}$ less sensitive to errors in $t_{1}$ and $t_{2}$. It should be noted, that the coefficient $C_{t / n}$ is specific for each channel.

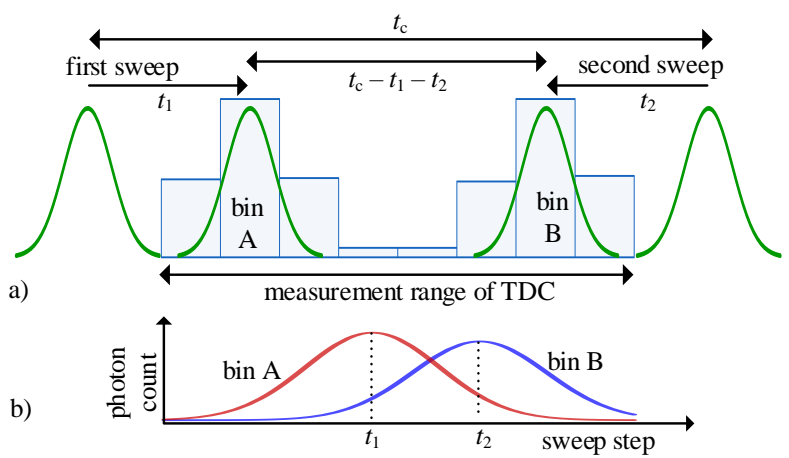

Fig. 2. a) Principle for setting the measurement range of TDC to absolute time scale, b) photon counts of selected bins A and B plotted against the sweep step.

\section{B. Simple Method for the Characterization of a Sensor}

A simple method for the characterization of a sensor is based on a single measurement of a reference sample with a short fluorescence lifetime. For each channel $k$, a weighted average $m_{k}$ of the histogram (average bin number weighted by photon counts) is calculated. As all channels receive a photon distribution of same shape (fluorescence of reference sample), for an ideal sensor the average value $m_{k}$ would be the same for every channel. For a real sensor, the average value $m_{k}$ varies due to differences of the channels and a variation of $m_{k}$ describes a dominant shape of the timing skew as shown in Fig. 3.

\section{TIMING SKEW COMPENSATION}

The basic idea of the timing skew compensation technique is shown in Fig. 4. Fig. 4 a) represents a sensor that has 100 channels, a TDC resolution of $100 \mathrm{ps}$ and no timing skew. With such an ideal sensor, the time gate endpoint can be set in every channel to e.g. 400 ps just by collecting photon counts from bins 1-4. Fig. 4 b) shows characteristics of the same kind of sensor with some added timing skew. If time gating is now done simply by collecting photon counts from bins $1-4$, the endpoint of the time gate will vary within $390-440$ ps causing distortion to the measured spectrum.

If a sensor is characterized with one of the methods mentioned above, a large part of the distortion can be eliminated by using the characterization data. If the precise method was used for the characterization, the compensation can be done as in Fig. 4 c). As an example, characterization data tell that in channel 60 , bin 4 covers the interval $306-416$ ps. To set the endpoint of the time gate effectively to $400 \mathrm{ps,} \mathrm{only} \mathrm{a} \mathrm{part} \mathrm{of} \mathrm{the}$ photon count from bin 4 should be included. In this case, the included part shown with green color in Fig. 4 c) is approximately (400 ps - $306 \mathrm{ps}) /(416 \mathrm{ps}-306 \mathrm{ps}) \approx 85 \%$. So, for channel 60 , the compensated photon count would be $n_{1}+n_{2}+$ $n_{3}+0.85 n_{4}$. The last term of the sum should be determined separately for each channel to achieve an effective endpoint of time gate at $400 \mathrm{ps}$ along the whole sensor.

When a sensor is characterized with the simple method, an average value $m_{k}$ of a histogram is calculated in bin numbers for each channel $k$. For a time gate constructed of bins $y$ to $x$, timing skew can now be compensated by adding a compensation term $\left(m_{k}-\min \left(m_{k}\right)\right) n_{x+1}$ to $\operatorname{sum} n_{y}+n_{y+1}+\cdots+n_{x}$, where $\min \left(m_{k}\right)$ is the minimum value of $m_{k}$.

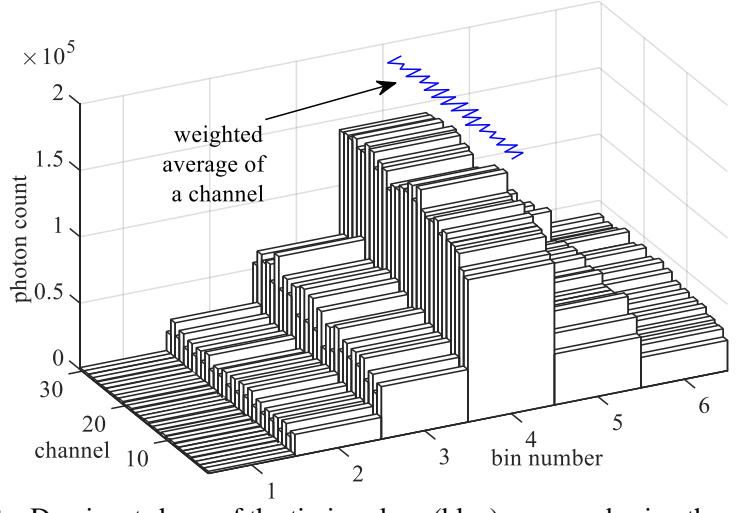

Fig. 3. Dominant shape of the timing skew (blue) measured using the simple characterization method. Only channels 1-30 are shown for clarity. 

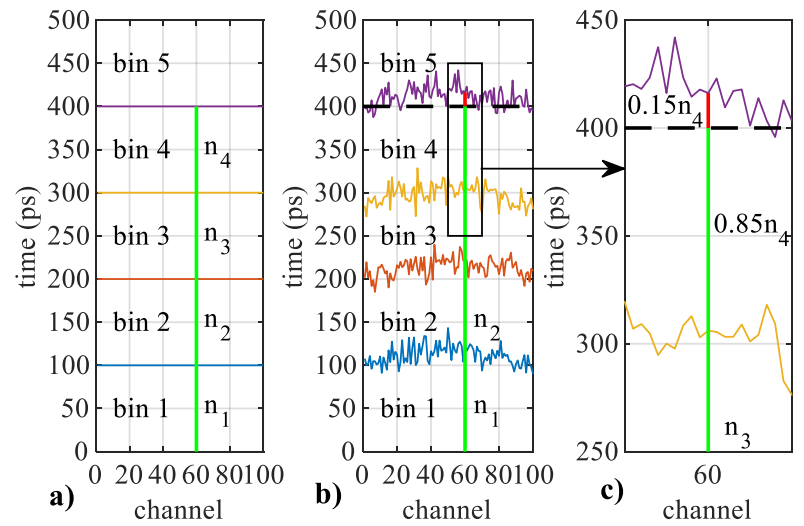

Fig. 4. Timing properties of a) an ideal sensor (no timing skew) and b) a sensor with timing skew, c) principle of the timing skew compensation, close-up zoom of channel 60 .

\section{Measurement Setur AND Results}

All measurements were made using a Raman spectrometer described in detail in [6]. Compared to the setup in [6], the laser was replaced with a new one having a pulse width, energy and rate of $150 \mathrm{ps}(\mathrm{FWHM}), 0.5 \mu \mathrm{J}$ and $350 \mathrm{kHz}$, respectively. The nominal resolution of the TDCs was set to about $100 \mathrm{ps}$. The reference sample used for the characterization was an aqueous solution of erythrosin B (Sigma-Aldrich no. 198269) having a short fluorescence lifetime of about 90 ps [9]. 256 channels of the sensor covered a spectral range of $220-1870 \mathrm{~cm}^{-1}$ but a spectral range of $800-1800 \mathrm{~cm}^{-1}$ (channels $85-244$ ) is shown in Figs. 5 and 6 as it is the most relevant part for sesame seed oil.

\section{A. Measurement Results for the Precise Method}

In the continuous light measurement, the sensor was illuminated with an incandescent lamp for 45 minutes. Sweeps to determine intervals $t_{1}$ and $t_{2}$ were made by changing the length of an adjustable coaxial cable (874-LAL) with $1 \mathrm{~mm}$ steps $(3.3373 \mathrm{ps} / \mathrm{mm})$. Bin 2 was used in the first sweep and bin 5 was used in the second sweep. For each step, one million laser pulses were shot. A polynomial was fitted to the sweep results, and polynomial values were used instead of measured photon counts to improve the accuracy to find $t_{1}$ and $t_{2}$. A long delay $t_{\mathrm{c}} \approx 804$ ps was induced by increasing the length of same coaxial cable by $241 \mathrm{~mm}$ at once.

Measured timing characteristics of the sensor are shown in Fig. 5 a), and distortion caused by the timing skew is significant in the uncompensated spectrum of sesame seed oil as shown in Fig. $5 \mathrm{~b}$ ) (red). Photon counts of bins 1-4 were summed to create the uncompensated spectrum. Timing skew compensation was made with a computational time gate at 550 ps shown with a black line in Fig. 5 a). When compensation is applied, most of the distortion disappears, as shown in Fig. 5 b) (blue). The ratio between distortion levels in uncompensated and compensated spectra is approximately $1: 4$.

\section{B. Measurement Results for the Simple Method}

In the simple characterization, ten million laser pulses were shot at the reference sample. The average values of the histograms $\left(m_{k}\right)$ and the compensation values $\left(m_{k}-\min \left(m_{k}\right)\right)$ for
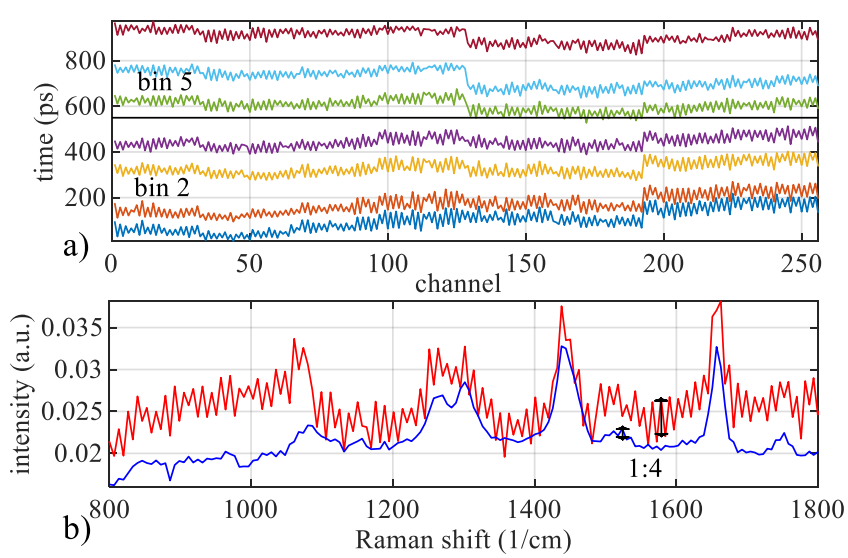

Fig. 5. a) Measured timing characteristics of a sensor, b) uncompensated (red) and compensated (blue) Raman spectrum of sesame seed oil.

each channel are shown in Fig. 6 a). In Fig. 6 b), the uncompensated spectrum (red) that consists of photon counts from bins $1-3$ is visibly distorted. For timing skew compensation, compensation terms were calculated by multiplying photon counts of bin 4 with compensation values shown in Fig. 6 a). The compensated spectrum was formed by adding photon counts from bins 1-3 and the compensation term together. In the compensated spectrum (blue) shown in Fig. 6 b), the level of distortion is significantly lower. The importance of compensation is visible particularly well around a small Raman peak at $1750 \mathrm{~cm}^{-1}$.

\section{CONCLUSIONS}

Depending on a sensor and a sample, timing skew of a line sensor may be the dominant error source in time-gated Raman spectroscopy. Two methods for determining the timing skew of a CMOS SPAD line sensor to compensate for its effects on the Raman spectrum were developed. With the presented methods, about $75 \%$ of the distortion caused by the timing skew could be removed and the signal-to-noise ratio of measured spectra could be significantly improved. Timing skew compensation is one way to improve the performance of time-gated Raman spectroscopy, especially when challenging (high fluorescence level, short fluorescence lifetime) samples are to be measured.
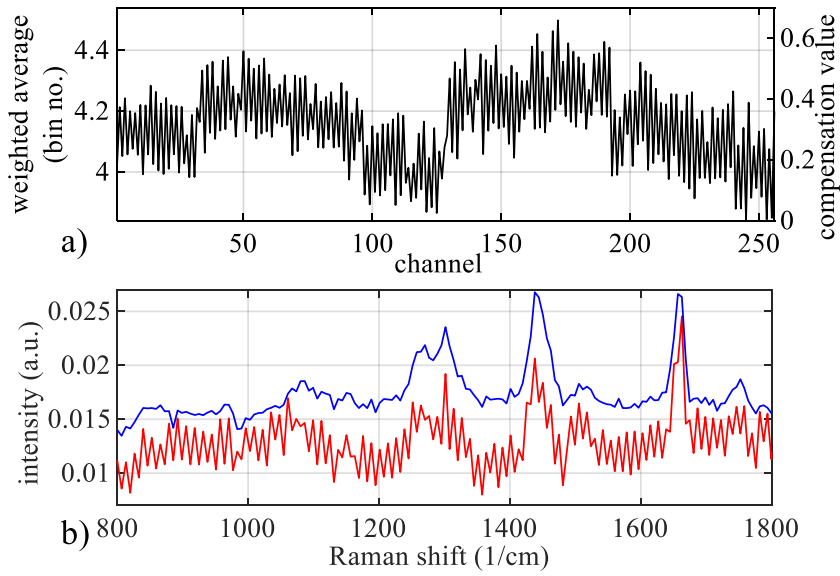

Fig. 6. a) Measured shape of the timing skew, b) uncompensated (red) and compensated (blue) Raman spectrum of sesame seed oil. 


\section{REFERENCES}

[1] R. P. Van Duyne, D. L. Jeanmaire, and D. F. Shiver, "Mode-locked laser Raman spectroscopy - A new technique for the rejection of interfering background luminescence signals," Analytical Chemistry, vol. 46, no. 2, pp. 213-222, 1974.

[2] K. Goluk et al., "Is photobleaching necessary for Raman imaging of bone tissue using a green laser?," Biochimica et Biophysica Acta, vol. 1758, pp. 868-873, 2006.

[3] A. Campion and P. Kambhampati, "Surface-enhanced Raman scattering," Chemical Society Reviews, vol. 27, pp. 241-250, 1998.

[4] I. Nissinen et al., "A sub-ns CMOS single photon avalanche diode detector for Raman spectroscopy," Proceedings of the European SolidState Device Research Conference (ESSDERC), pp. 375-378, 2011.

[5] J. Blacksberg, Y. Maruyama, E. Charbon, and G. R. Rossman, "Fast single-photon avalanche diode arrays for laser Raman spectroscopy," Optics Letters, vol. 36, no. 18, pp. 3672-3674, 2011.
[6] I. Nissinen, J. Nissinen, P. Keränen, D. Stoppa, and J. Kostamovaara, "A 16x256 SPAD line detector with a 50-ps, 3-bit, 256-channel time-todigital converter for Raman spectroscopy,” IEEE Sensors Journal, vol. 18, pp. 3789-3798, 2018.

[7] A. Usai, N. Finlayson, C. D. Gregory, C. J. Campbell, and R. K. Henderson, "Separating fluorescence from Raman spectra using a CMOS SPAD TCSPC line sensor for biomedical applications," Proc. SPIE 10873, Optical Biopsy XVII: Toward Real-Time Spectroscopic Imaging and Diagnosis, 2019.

[8] J. Holma, I. Nissinen, J. Nissinen, and J. Kostamovaara, "Characterization of the timing homogeneity in a CMOS SPAD array designed for timegated Raman spectroscopy," IEEE Transaction on Instrumentation and Measurement, vol. 66, no. 7, pp. 1837-1844, 2017.

[9] N. Boens et al., "Fluorescence lifetime standards for time and frequency domain fluorescence spectroscopy," Analytical Chemistry, vol. 79, pp. 2137-2149, 2007 